\title{
Formulation Optimization, Rheological Characterization and Suitability Studies of Polyglucoside-based Azadirachta indica A. Juss Emollient Cream as a Dermal Base for Sun Protection Application
}

\author{
KAUSHITA BANERJEE, N. THIAGARAJAN ${ }^{1}$ AND PADMA THIAGARAJAN* \\ School of Biosciences and Technology, VIT University, Vellore- 632 014, ${ }^{1}$ Material Science Division, National Aerospace \\ Laboratories, Bangalore-560 017, India
}

Banerjee, et al.: Sun Protective Neem Emollient Cream

\begin{abstract}
Polyglucoside emulsifiers have been very beneficial for blending natural oils with water for formulation of emollient creams that can serve as effective topical applicants. In the present study, the formulation process for neem (Azadirachta indica A. Juss) oil emollient cream was optimized by phase inversion method in terms of reduced particle size with respect to ultrasonication conditions like duty cycle, time, cavitation intensity and amplitude. Its diluted variant and the one dispersed with titanium dioxide nanoparticles were also synthesized and comparisons of mean particle size, zeta potential and electrophoretic mobility were made with three popular commercial cosmetic creams. Rheological studies showed similar results with respect to relevant parameters like hysteresis loop areas, yield stress, consistency and pseudoplasticity indices. All creams showed non-Newtonian thixotropic behaviour and shear thinning feature that contribute to good flow characteristics and favourable spreading ability. The sun protection factor, determined for the titanium dioxide-loaded cream was found to be better than a popular commercial cream. It was also found to be harmless to representative skin flora as determined by disc diffusion method. Cell toxicity studies on $3 T 3$ cell lines carried out for the oil and emulsifier showed the cells to be viable in the presence of these components. It is thus inferred that this neem emollient base cream can be safely used as a cosmetic topical applicant for skin protection.
\end{abstract}

Key words: Alkyl polyglucoside, Azadirachta indica, rheograms, sun protection factor, titanium dioxide

Emollient creams can serve as base matrices and vehicles to transport active ingredients into biological systems, by the topical route of delivery. Emollients possess optical clarity, a large surface area, are easy to prepare and thermodynamically and kinetically stable. Their transdermal usage is non-invasive, patient compliant and susceptible to self-administration. They can serve as an effective replacement for delivery of pharmaceuticals and cosmeceuticals by the oral and mucosal routes ${ }^{[1,2]}$, wherein the first-pass effect of liver, that leads to untimely metabolism of active compounds can be avoided. In addition, emollients also facilitate sustained release of loaded active compounds through the dermis and epidermis as their formulations remain intact on the skin and continue to deliver them in a controlled manner over long periods of time ${ }^{[3]}$. Cosmeceuticals, loaded onto topical emollients have both therapeutic and occlusive impacts on the skin

*Address for correspondence E-mail: padmadk4@gmail.com

November-December 2017 wherein they achieve healing and appealing effects ${ }^{[4]}$. They also prevent ultraviolet damage, minimize free radical formation and provide it with a smooth and uniform texture. Dosage forms for dermatological remedies are intended to promote restorative and curative action at specific sites in the epidermis.

However, many such cosmeceutical-based emollient creams like facial moisturizers, shampoos, antiaging and shaving creams and sunscreen lotions contain synthetic chemicals as preservatives and stabilizing agents. They may have several toxic and deleterious

This is an open access article distributed under the terms of the Creative Commons Attribution-NonCommercial-ShareAlike 3.0 License, which allows others to remix, tweak, and build upon the work non-commercially, as long as the author is credited and the new creations are licensed under the identical terms

Accepted 17 September 2017

Revised 20 March 2017

Received 14 November 2016

Indian J Pharm Sci 2017;79(6):914-922 
effects that have been reported, documented and debated $^{[5,6]}$. In this context, it is anticipated that plant oil-based emollient creams, formulated with biobased emulsifiers, could serve as excellent matrices for supporting several cosmeceutical ${ }^{[7]}$. Such formulations offer advantages of products with green chemistry possibly with low adverse dermatological effects. The natural carotenoids, tocopherols and other antioxidants present in them dispense requirements for extraneous addition of synthetic compounds for their preservation and stability to a large extent.

Synthesis and characterization of such a neem oil emollient cream based on a polyglucoside emulsifier, with a promising potential for dermatological applications, has earlier reported by us ${ }^{[8]}$. As a further development, in the present study, the optimization of the formulation procedure, in terms of oil:emulsifier ratio, maltase cross formation and particle size of the emollient cream under variable ultrasonication conditions like duty cycle (DC), sonication time, cavitation intensity and amplitude, have been standardized to get a best possible base matrix with respect to reduced particle size. Rheological properties of this cream, its diluted variant and one loaded with titanium dioxide $\left(\mathrm{TiO}_{2}\right)$ nanoparticles have been assessed with respect to related parameters like yield stress, pseudoplasticity and consistency indices, to determine its suitability, in comparison with similar commercially marketed popular neem oil products. The sun protection factor (SPF) of the cream loaded with $\mathrm{TiO}_{2}$ nanoparticles (essential component and whitening agent in sunscreens) has been measured and compared with a commercially available sun blocking cream (SPF15). Its antibacterial activity against certain normal skin biota has also been tested to report its suitability for use as a topical applicant. Percentage viability of 3T3 (keratinocytes) cell lines in the presence of the oil and emulsifier, at concentrations used in the emollient cream, has been checked as a part of toxicity assessment studies.

\section{MATERIALS AND METHODS}

Neem oil was purchased from Falcon Essential Oils, Bangalore, India. Alkylpolyglucoside emulsifier, marketed under the trade name of Montanov 202 was procured from Yasham Distributers, Mumbai. $\mathrm{TiO}_{2}$ nanoparticles were obtained from SRL Chemicals. Analytical grade ethanol, from Sigma Aldrich, was used for SPF measurements, in ratio of 40:60 with water. All bacterial media components were from HiMedia Pvt.
Ltd. Ayur Herbal Moisturizer (CC1), Dove body lotion (CC2), Boroplus moisturizing lotion (CC3), and Nivea Whitening Cell Repair cream (CC4) were purchased from the local market. MilliQ water was used for all experiments. Bacterial skin flora representatives were isolated from human volunteers by swabbing the skin and the organisms were cultured onto nutrient agar plates.

\section{Instruments and statistical analysis:}

Ultrasonicator (Sonics Vibra cell with ultrasonic frequency of $20 \mathrm{kHz}$, with probe diameter of 13 $\mathrm{mm}$ ), Horiba Scientific SZ 100 particle size analyzer (scattering angle of $90^{\circ}$ at $25^{\circ}$ ) with Windows [Z type] version 2.00 software, Systronics double beam UV/Vis Spectrophotometer (AU 2701) equipped with $1 \mathrm{~cm}$ quartz cell and Brookfield DV2T Viscometer (Spindle no. LV-4 (64) and sample chamber SC413RPY with temperature probe and cable) were used for the experiments. Microplate reader Biorad 680 was employed for cell culture studies.

All experiments were carried out in triplicates and values were expressed as mean $\pm \mathrm{SD}$. One way analysis of variance (ANOVA, $\mathrm{P}<0.05$ ) was then used, along with Tukey's test, wherever applicable to arrive at significant differences between the experimental values $^{[9]}$.

\section{Emollient cream formulation:}

Five grams of oil was weighed into a series of conical flasks and were warmed to $75^{\circ}$. Then, 0.5 to $5 \mathrm{~g}$ of emulsifier, in increments of $0.5 \mathrm{~g}$, were added to each of these flasks and mixed well. Water maintained at $75^{\circ}$ was added in drops, with constant stirring, to all flasks to make up the total volume to $50 \mathrm{ml}$. The best oil:emulsifier ratio was fixed by visually observing the non-separation of oil or non-dissolution of emulsifier in each of the flasks. A drop of formulations, on glass slides were observed under light microscope at 40x for Maltese cross formation.

Based on these observations, the formulation with oil:emulsifier ratio of 1:0.4 was selected for optimizing the ultrasonication parameters at a pulse rate of $2 / \mathrm{min}$ to achieve maximum possible reduction in particle size, measured $24 \mathrm{~h}$ after synthesis, under these conditions. DC (3.33, 6.66, 9.99, 13.33 and $16.66 \%)$, ultrasonication time $(3,5,7,9$ and $11 \mathrm{~min})$, cavitation intensity $(75.41,150.82,226.24,301.65,377.07$ $\left.\mathrm{W} / \mathrm{cm}^{2}\right)$ and amplitude of sonication $(30,40,50,60$, $70 \%$ ) were standardized serially one after the other in 
this order by using previously reported standard values for synthesis of such formulations for optimization of the preceding parameters ${ }^{[10]}$. In each case, the minimum statistically significant particle size was the criterion for selection of the best value.

The final emollient cream (EC1) was then synthesized under the completely optimized conditions. It's doubly diluted variant (with $50 \mathrm{ml}$ of MilliQ water, EC2), was also synthesized for comparison of rheological properties. Additionally, $1 \% \mathrm{TiO}_{2}$ nanoparticles were dispersed in $50 \mathrm{ml}$ of EC1 (represented as EC3) for further analysis. Particle size, zeta potential and electrophoretic mobility were measured for all creams including the commercial ones.

\section{Rheology studies:}

Data, in terms of torque and apparent viscosity, at rpm ranging from 5 to 200 was obtained at $37^{\circ}$ for all cream. Shear rate (1.09/s to $43.95 / \mathrm{s})$ and stress were calculated using specific "System Factors" based on sensor system geometry. Dynamic shear rate mode, was operated in ascending and descending orders, and plotted against shear rate, for obtaining the hysteresis loop and its area was calculated. The plot of their corresponding logarithmic components was used to obtain yield stress by extrapolation. Similarly, log viscosity was plotted against log shear rate to obtain values of consistency indices $(\mathrm{k})$, again by extrapolation. The slope gave the pseudoplasticity indices $(\mathrm{PI})^{[11,12]}$.

\section{Determination of in vitro SPF:}

The procedure described by Sudhahar and Balasubramanian was used for this determination ${ }^{[13]}$. The absorbance values of cream solutions were measured from 290 to $320 \mathrm{~nm}$ at $5 \mathrm{~nm}$ intervals against ethanol: water mixture. SPF was calculated by using the following Eqn. ${ }^{[14]}: \mathrm{SPF}=\mathrm{CF} \times \sum_{290}{ }^{320} \times \mathrm{EE}(\lambda) \times \mathrm{I}(\lambda) \times \operatorname{Abs}(\lambda)$, where, $\mathrm{CF}=$ correction factor $(10), \mathrm{EE}(\lambda)=$ erythmogenic effect of radiation with wavelength $\lambda, \operatorname{Abs}(\lambda)=$ optical density at wavelength $\lambda$. The values of $\mathrm{EE} \times \mathrm{I}$ are constants.

\section{Isolation and activity against skin flora:}

Skin bacteria was swabbed from volunteers after obtaining informed consent using sterile cotton swabs, spread on nutrient agar plates and incubated for $24 \mathrm{~h}$ at $37^{\circ}$. The isolated colonies after Gram staining and recording of morphological characteristics, were then sub cultured onto separate agar slants and biochemical tests were conducted for identification using standard protocols $^{[15]}$. Antibacterial activity of EC1 against these (0.5 McFarland scale), was assessed by Kirby Bauer disc diffusion method, $48 \mathrm{~h}$ after their preparation and after soaking the discs in the cream for $6 \mathrm{~h}$. Sterile water was used as negative control and respective antibiotic discs were used as positive controls. The plates were incubated at $37^{\circ}$ for $24 \mathrm{~h}$ and were checked for zones of inhibition ${ }^{[16]}$.

Cell viability studies of $3 \mathrm{~T} 3\left(1 \times 10^{5}\right.$ cells/well $)$ cell lines in presence of 5,10 and $15 \%$ of neem oil and 1,2, $5 \%$ of emulsifier separately in $10 \%$ dimethyl sulfoxide (DMSO) was assessed by standard methotrexate (MTT) assay ${ }^{[17]}$. Spectrophotometric absorbance of the purple blue formazan dye was measured at $570 \mathrm{~nm}$ and the cytotoxicity percentage was plotted using graph pad prism 5 software for each concentration ${ }^{[18]}$. The corresponding photomicrographs were also captured.

\section{RESULTS AND DISCUSSION}

Polyglucosides belong to a novel class of non-ionic emulsifiers that have been known for more than a century ${ }^{[19]}$ but due to limitations in their large-scale production, they were largely underexploited. Recently, their commercial production, from renewable and regrowable resources like vegetable raw materials like corn, coconut oil, manioc and potato, have become feasible. Their chemical structures, with strong hydrophilic glucose residues and alcohol residues as hydrophobic parts, serve as excellent emulsifying agents for green formulations with natural vegetable oils. They have excellent dermatological compatibility, biodegradability and ecological acceptability and are also conducive to modifications of rheological properties of topical applicants. They act as liquid crystal reservoirs to improve hydration and form elastic networks with water. Hence overall stability of their formulations and long-term maintenance of skin moisture is achieved ${ }^{[20]}$.

The preparation method has a pivotal role to play in the emulsification process. Thus, phase inversion emulsification has been adopted for the synthesis of the emollient cream. This method has been widely employed in development of cosmeceutical, pharmaceutical and nutraceutical products. Phase inversion is best described when adequate agitation to the components of oil in water emulsion, reverts to water in oil and vice versa. This leads to very low particle sizes which in turn confer overall stability of the formulation. Emulsification using this advanced technique results in the emergence of extremely fine droplets dispersed in the continuous phase. Traditional emulsification processes, on the other hand, is responsible for the 
formation of unstable macroemulsions with increased particle sizes due to coalescence, as the emulsifier is dissolved into the continuous phase and then added to the dispersed phase under appropriate agitation. Thus, in phase inversion method, the emulsifier in the system migrates from the bulk to the interface inducing "Marangoni stress" due to low interfacial tension. The Marangoni effect decelerates the expulsion of the continuous phase film between two opposing droplets (an essential step leading to coalescence) and is important phenomena to understand the emulsion morphology structure ${ }^{[21]}$.

A ratio of 1:0.4 of neem oil:polyglucoside emulsifier was found to produce a Maltese cross structure (fig. 1) with hydrated liquid crystals that are arranged in a crystalline fashion to link the oil and emulsifier molecules. This stable interlinking builds an intermediate molecular ordering between the excess emulsifier, and stabilized oil droplets that are represented as interlinked crystals. This structural phenomenon confers additional stability to the system and also aids efficient lubrication upon topical application $^{[22,23]}$.

Fig. 2a-d shows the trend in the average particle size of the EC1 emollient cream with variations in DC, sonication time, cavitation intensity and amplitude, respectively. The least particle size $(403.8 \pm 6.4 \mathrm{~nm})$ was got for a DC of $16.66 \%$. So this percentage (stop time of $5 \mathrm{~s} / 30 \mathrm{~s}$ ) was selected as it saves maximum energy during sonication and hence is more economical. During sonication, a time of 5 min gave a size average of $417.6 \pm 4.9 \mathrm{~nm}$ and at longer times, the sizes were

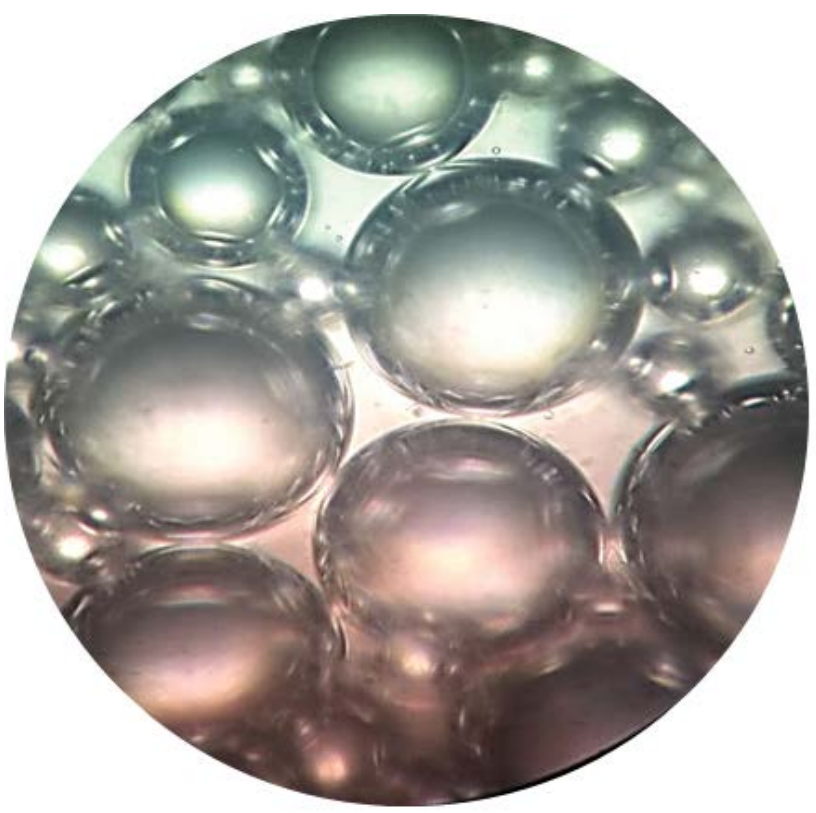

Fig. 1: Maltese cross formation in neem oil emollient cream
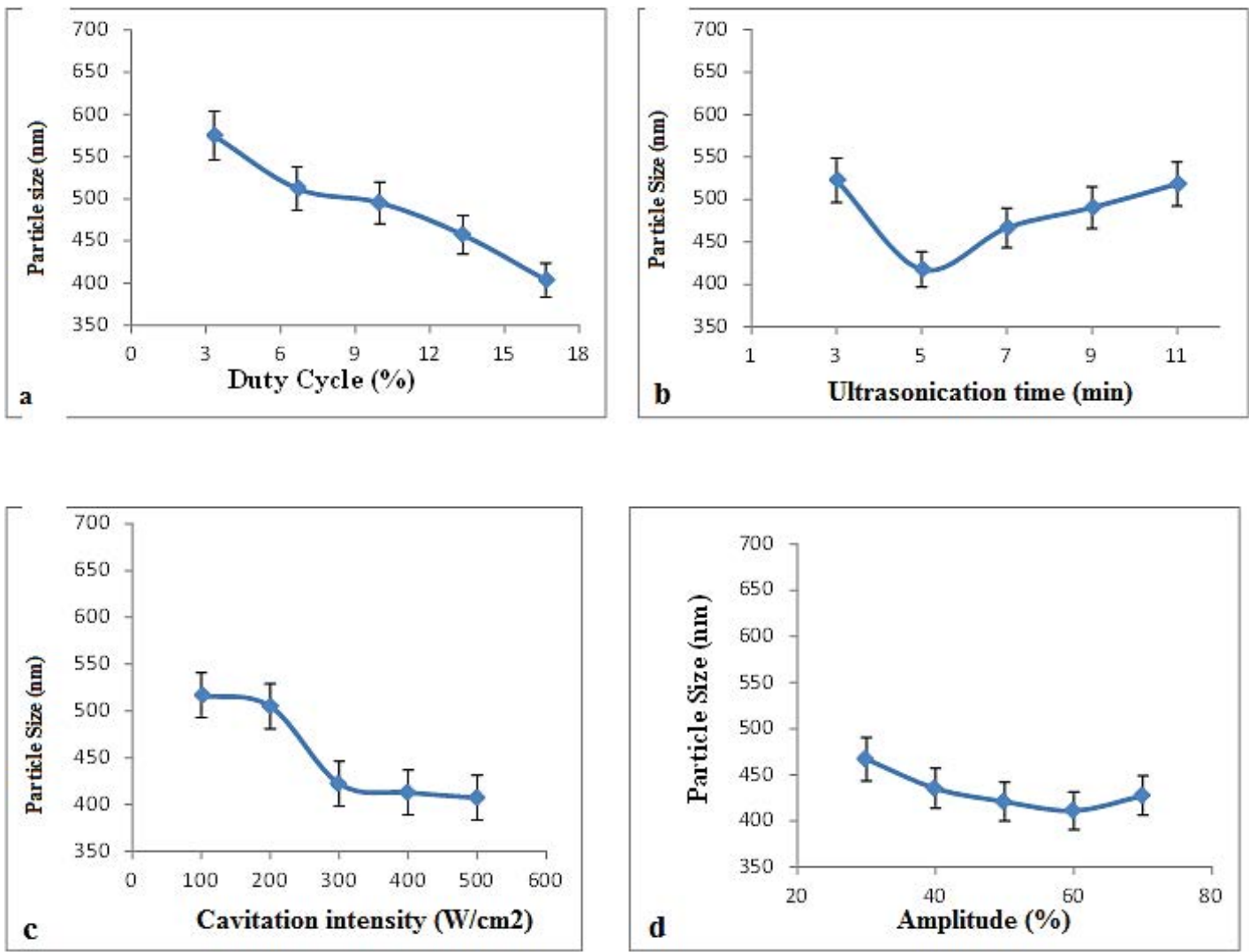

Fig. 2: Effect of various parameters on particle size of neem oil emollient cream a: Duty cycle, b: ultrasonication time, c: cavitation intensity, $d$ : amplitude; $M e a n \pm S D$ for $n=3$ 
higher. It is relevant to state here that size reduction, achieved during the ultrasonication, is only during the "pulse off" period. In the "pulse on" period, the particles keep growing and when it is in off mode, they collapse with high increase in local temperatures, under the influence of which the particles break down and their size is reduced. However, this happens only if the growing sizes are in the active range. If the sizes are higher, then efficient size reduction is not achieved as seen in the cases of higher sonication times beyond 5 min wherein particles achieve a higher initial size during "pulse on" period and these particles upon collapse during the off period are not able to bring about a substantial reduction in their sizes. For cavitation intensity, minimum particle size of $423.1 \pm 12.8 \mathrm{~nm}$ was at $226.24 \mathrm{~W} / \mathrm{cm}^{2}$. Sizes for higher intensities were not significantly lower and hence this value was selected to save energy. Previous reports have stated that intensities with power inputs between 300 and $450 \mathrm{~W}$ are ideal for of non-Newtonian fluid emulsification ${ }^{[10]}$. Amplitude values between 30 and $70 \%$ produced particles sizes with no significant difference. Hence $50 \%$ was chosen as amplitudes between 45 and $65 \%$ are most suitable for efficient emulsification ${ }^{[24]}$.
Particle size, polydispersity index, zeta potential and electrophoretic mobility of the optimized emollient cream EC1, DC: $16.66 \%$; time: $5 \mathrm{~min}$; intensity: $226.24 \mathrm{~W} / \mathrm{cm} 2$; amplitude: 50\%), and also EC2 and EC3 are shown in Table 1. Fig. 3a and b represent graphical outputs of particle size and zeta potential for EC1. Formulations with zeta potentials above $+30 \mathrm{mV}$ and below $-30 \mathrm{mV}$ are most stable ${ }^{[25]}$ with respect to particle size and in this context; this emollient cream is expected to be very stable.

Rheology studies, viscosity values of EC1, EC2 and EC3 ranged from $250(5 \mathrm{rpm})$ to $13 \mathrm{~Pa}(200 \mathrm{rpm})$. The commercial creams were subsequently chosen to encompass these values $(350 \mathrm{~Pa}$ at $5 \mathrm{rpm}$ and $10 \mathrm{~Pa}$ at $200 \mathrm{rpm}$ ) in order to assess the suitability of using these emollient creams as a structural substitute for the commercial ones. Non-Newtonian behaviour of all creams can be inferred from their ascending shear rate line (fig. 4a). The lines are characterized by small linear trends at very low and high shear rates, with a non-linear power law region in between and follows the Ostwald-de Waele equation ${ }^{[26]}$. This model is apt for describing material flow behaviour under a small range of shear rates as used in our experiments. The

\section{TABLE 1: EVALUATION OF EMOLLIENT CREAMS (EC1-EC3) AND COMMERCIAL CREAMS (CC1-CC3)}

\begin{tabular}{|c|c|c|c|c|c|c|c|c|}
\hline Parameters & & & & & & & & \\
\hline Cream type & $\begin{array}{l}\text { Particle } \\
\text { Size }(\mathrm{nm})\end{array}$ & $\begin{array}{l}\text { dispersity } \\
\text { index }\end{array}$ & $\begin{array}{l}\text { Potential } \\
(\mathrm{mV})\end{array}$ & $\begin{array}{l}\text { phorectic } \\
\text { mobility } \\
(\mu \mathrm{mcm} / \mathrm{Vs})\end{array}$ & $\begin{array}{l}\text { hysteresis } \\
\text { loop (kPa.s) }\end{array}$ & $\begin{array}{l}\text { Yield stress } \\
\quad(\mathrm{Pa})\end{array}$ & $\begin{array}{c}\text { Consistency } \\
\text { index }\end{array}$ & $\begin{array}{l}\text { plasticity } \\
\text { index }\end{array}$ \\
\hline EC1 & $430.4 \pm 23.0$ & $1.10 \pm 0.20$ & $-71.4 \pm 3.2$ & $-3.335 \pm 0.27$ & $215.7 \pm 6.74$ & $3.4 \pm 0.2$ & $238.3 \pm 51.1$ & $0.4 \pm 0.06$ \\
\hline EC2 & $299.8 \pm 13.0$ & $0.80 \pm 0.10$ & $-64.4 \pm 1.20$ & $-3.70 \pm 0.06$ & $101.3 \pm 3.95$ & $3.02 \pm 0.05$ & $80.4 \pm 10.2$ & $0.5 \pm 0.07$ \\
\hline EC3 & $469.1 \pm 8.9^{*}$ & $1.20 \pm 0.30$ & $-77.7 \pm 4.60^{*}$ & $-4.020 \pm 1.40$ & $185.1 \pm 13.0$ & $3.5 \pm 0.04^{*}$ & $240.7 \pm 25.7$ & $0.4 \pm 0.03$ \\
\hline CC1 & $445.5 \pm 11.0$ & $0.80 \pm 1.30$ & $-68.3 \pm 1.30$ & $-3.120 \pm 1.50$ & $125.9 \pm 7.88$ & $3.29 \pm 0.1$ & $135.5 \pm 40.5$ & $0.6 \pm 0.01$ \\
\hline $\mathrm{CC} 2$ & $460.8 \pm 29.0^{*}$ & $0.87 \pm 0.38$ & $-70.1 \pm 2.40$ & $-3.361 \pm 0.90$ & $336.3 \pm 2.66$ & $3.6 \pm 0.3^{*}$ & $393.4 \pm 1838^{*, * *}$ & $0.7 \pm 0.1$ \\
\hline $\mathrm{CC} 3$ & $412.3 \pm 17.0$ & $1.0 \pm 2.240$ & $-65.7 \pm 3.60$ & $-3.40 \pm 1.10$ & $234.9 \pm 87.0$ & $3.3 \pm 0.005$ & $226.3 \pm 1112$ & $0.5 \pm 0.2$ \\
\hline
\end{tabular}

Statistically significant differences from EC2 and CC1 are represented as * and ** respectively

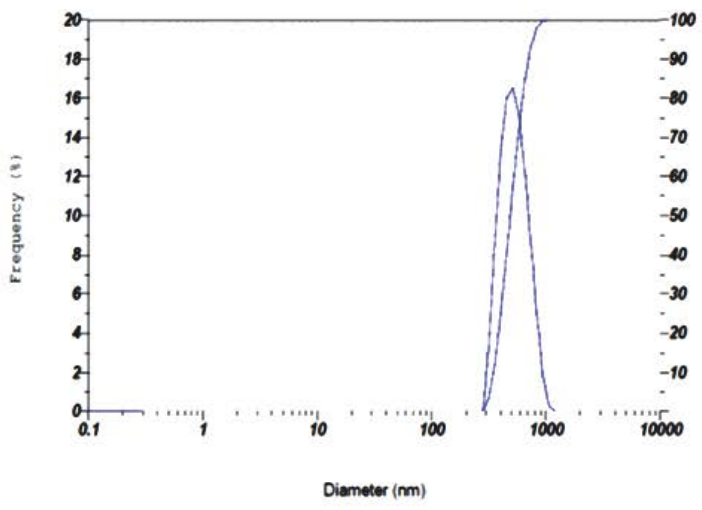

a

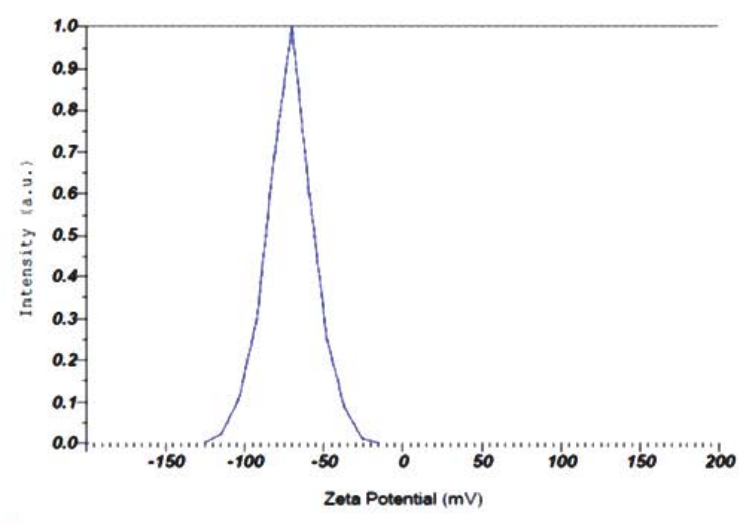

b

Fig. 3: Electrophoretic mobility analysis of EC1 a: Particle size, b: zeta potential 
results also indicate their pseudoplastic behaviour wherein they flow instantly upon increasing stress application thus attesting their shear thinning property. This dynamic study also reveals the capacity of these creams to achieve complete breakdown of their internal structures so that a smooth flow is obtained across the skin as shear stress is increased. When the stress is brought down slowly, the structures rebuild, the creams stick to the skin and do not continue to flow over it. This recovery behaviour is measured in terms of the areas of hysteresis loops and depicts their thixotropic behaviour ${ }^{[27]}$. The areas for all creams range from $215.7 \pm 6.764$ to $336.3 \pm 2.616 \mathrm{kPa} / \mathrm{s}$ (Table 1). The values are not significantly different showing similar thixotropic properties of the commercial creams and all variants of emollient creams formulated in this study. Previous reports have also shown the areas for hysteresis loops to be in the range of $118.62 \mathrm{kPa} / \mathrm{s}$ to $321.65 \mathrm{kPa} / \mathrm{s}$ and these are comparable with our values $^{[28]}$.

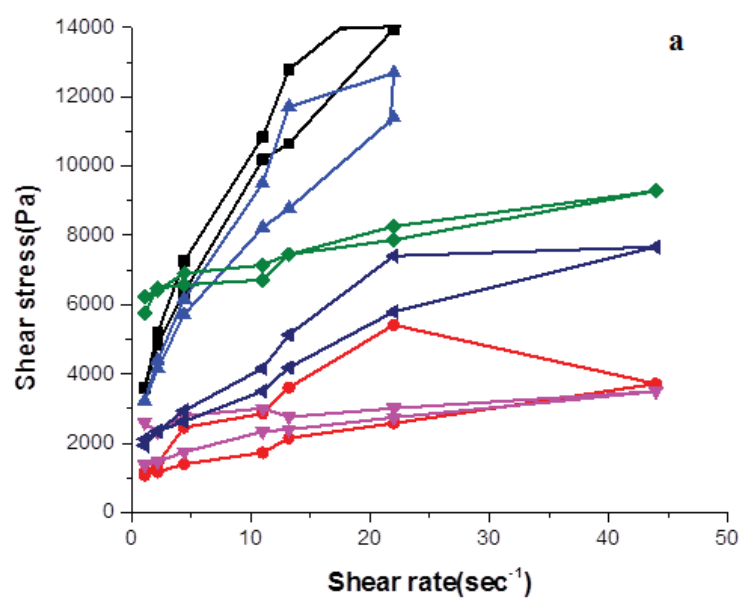

Yield stress represents the force that needs to be applied to make topicants spread effectively upon rubbing and also make a structured fluid flow. From the log-log plot of shear stress against shear rate (fig. 4b), it can be seen that yield stress for all creams range between $3.00 \pm 0.005$ to $3.60 \pm 0.30 \mathrm{~Pa}$ (Table 1). Several cosmetic products have yield stresses between 30 and $150 \mathrm{~Pa}$ suggesting the need for application of considerable amount of pressure for effective flow and spreading. In case of creams used here, they can flow and spread under very low dermal pressure ${ }^{[29}$. In case of EC1, EC2 and EC3, such low yield stress values also highlight the effectiveness of the polyglucoside emulsifier in trapping considerable amount of depot water in their internal structures. By soft rubbing, this depot water is released onto the skin ensuring better flow.

Log-log plot of viscosity against shear rate showed the consistency indices represented as " $\mathrm{k}$ " for all creams to be between $80.4 \pm 10.2$ for $E C 2$ and $393.4 \pm 183.8$ for CC2 
(fig. 4c and Table 1). Apparent viscosity (CI) predicts the texture and the constancy of the material. It is also an indicative of how efficaciously formulations are able to prevent the consecutive interglobular collisions, which are often possible reasons for creaming and oil separation over a period of time. From the slope of the same plots, PI for all creams was calculated to be in the range of $0.4 \pm 0.06$ to $0.7 \pm 0.1$ (Table 1). PI represents the extent of non-Newtonian fluid behaviour with values ranging from 0 to 1 . It is inversely proportional to pseudoplasticity of fluids and is a measure of their thinning property ${ }^{[30]}$. A unique combination of high $\mathrm{k}$ and low PI represent its time independent behaviour and ensures good dermal spreadability.

The determination of in vitro SPF using spectrophotometric method can be affected by factors like solvents, concentration of each active component, nature of base emollients, interaction and combination of diluents with emollient bases and skin and $\mathrm{pH}$ of creams. All these factors may result in variable UV absorption. For effectual prevention of UV radiation, creams must exhibit a wide range of absorbance between $290 \mathrm{~nm}$ and $400 \mathrm{~nm}$. The UV/Vis spectrophotometric method for this determination is easy, rapid and economical. It can be an alternative for in vivo determination of SPF values in many cosmetic formulations ${ }^{[13]}$. In the present study, EC1 and EC3 creams showed a SPF of 3.257 and 5.055 respectively, inferring that these can be used to achieve a considerable UV blockage, hence serving as potent cosmeceutical agents. SPF of the commercial sun blocking cream (labelled SPF 15) was found to be only 4.560 (Table 2).

In case of activity against normal skin flora, the representative normal skin bacteria isolated and identified at genus level here were Bacillus sp.,

\begin{tabular}{|c|c|c|c|c|}
\hline \multirow{2}{*}{$\begin{array}{l}\text { Wave } \\
\text { length } \\
(\lambda \mathrm{nm})\end{array}$} & \multirow{2}{*}{$\begin{array}{c}\mathrm{EE} \times \mathrm{I} \\
\text { (normalized) }\end{array}$} & \multicolumn{3}{|c|}{ Abs. $\times$ CF $\times E E \times I$} \\
\hline & & EC1 & EC3 & CC4 \\
\hline 290 & 0.015 & 0.0057 & 0.06345 & 0.09525 \\
\hline 295 & 0.0817 & 0.03758 & 0.360297 & 0.1152 \\
\hline 300 & 0.2874 & 1.2933 & 1.060506 & 1.36515 \\
\hline 305 & 0.3278 & 0.13112 & 1.235806 & 2.34377 \\
\hline 310 & 0.1864 & 1.08112 & 1.710593 & 0.4921 \\
\hline 315 & 0.0839 & 0.5926 & 0.521451 & 0.11802 \\
\hline 320 & 0.0817 & 0.11592 & 0.10368 & 0.02628 \\
\hline SPF & & 3.257 & 5.055 & 4.56 \\
\hline
\end{tabular}

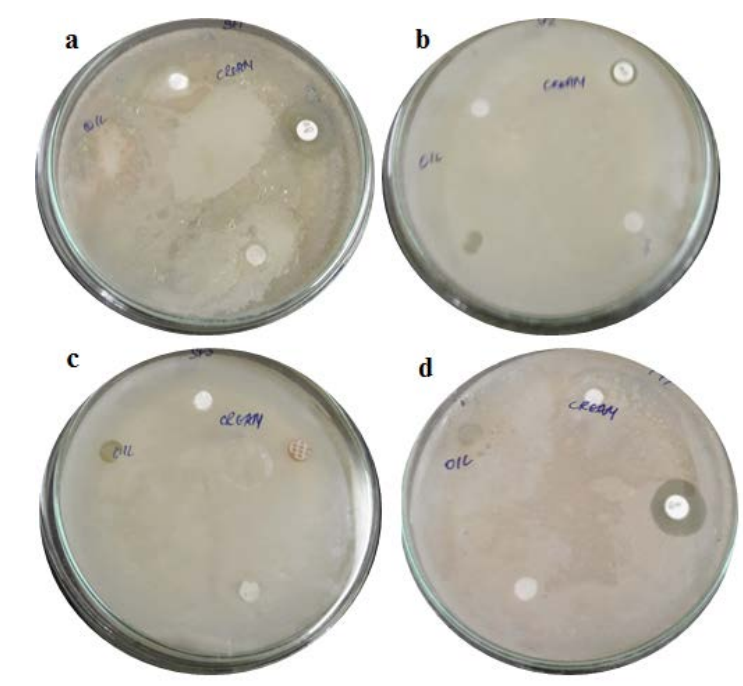

Fig. 5: Antibacterial activity of EC1 against normal skin flora a: Bacillus sp.; b: Staphylococcus sp.; c: Micrococcus sp.; d: Pseudomonas sp.

Staphylococcus sp., Micrococcus sp. and Pseudomonas sp. EC1 cream, tested on these bacteria, did not show any zone of inhibition (fig. 5) attesting its safe nature for application onto the skin. It is evident here that the emulsifier molecules containing glucoside moieties can hold water in them and mimicking the stratum corneum layer. Thus, the low molecular weight and water-attracting properties of such emulsifiers are essential in penetrating the epidermis of the skin by taking up water from the dermis region, and trapping it, therefore giving an occlusive impact on the skin without harming the beneficial bacteria present on the skin surface ${ }^{[16]}$.

Cell culture studies, the results of testing different concentrations of neem oil and emulsifier on the percentage viability of 3T3 cell lines are shown in the form of bar graphs are shown in fig. 6a and b, the pictomicrographs are depicted in (fig. 7a-g). Cytotoxicity of the control and test samples is measured by a concentration-dependent reduction in dye uptake by the cells after exposure to a test material. A decrease in the uptake of dye in treated cell cultures, following a test chemical exposure, is used to determine relative toxicity and viability. The results here reveal $95.77 \%$ of cell viability for oil and $96.15 \%$ for emulsifier thus concluding that each component incorporated in our cream formulations are safe to use on dermal cells.

Polyglucoside emulsifiers used for emulsifying neem oil, in this study, to produce emollient creams have good comparability in terms of rheology, with similar neem based formulations that are commercially available. Their properties, in terms of dermal requirements, as 

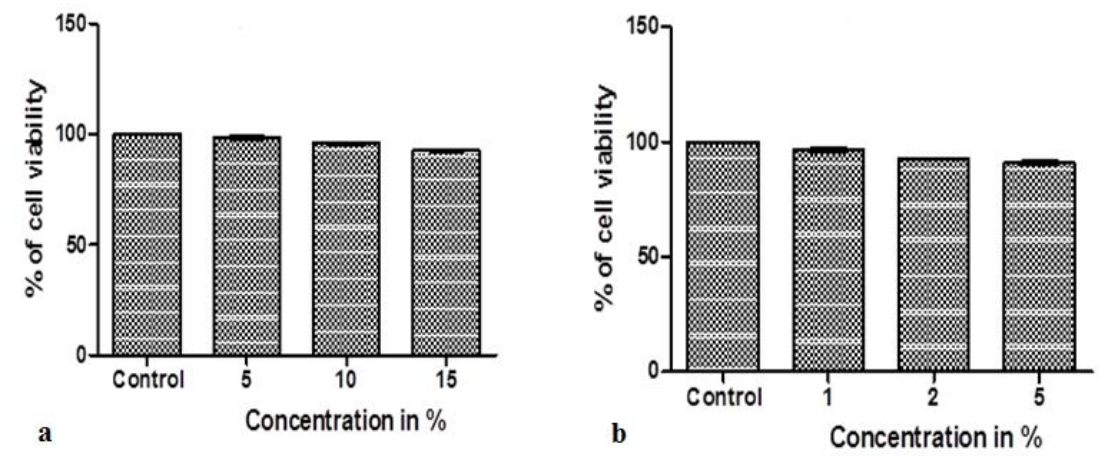

Fig. 6: Trends in percentage viability of $3 T 3$ cells assessed with different concentrations of neem oil and APG emulsifier a: Neem oil and b: APG emulsifier

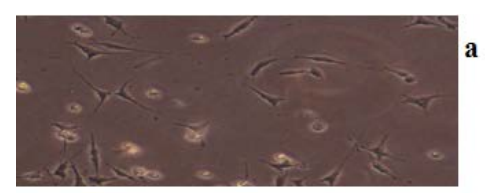

b
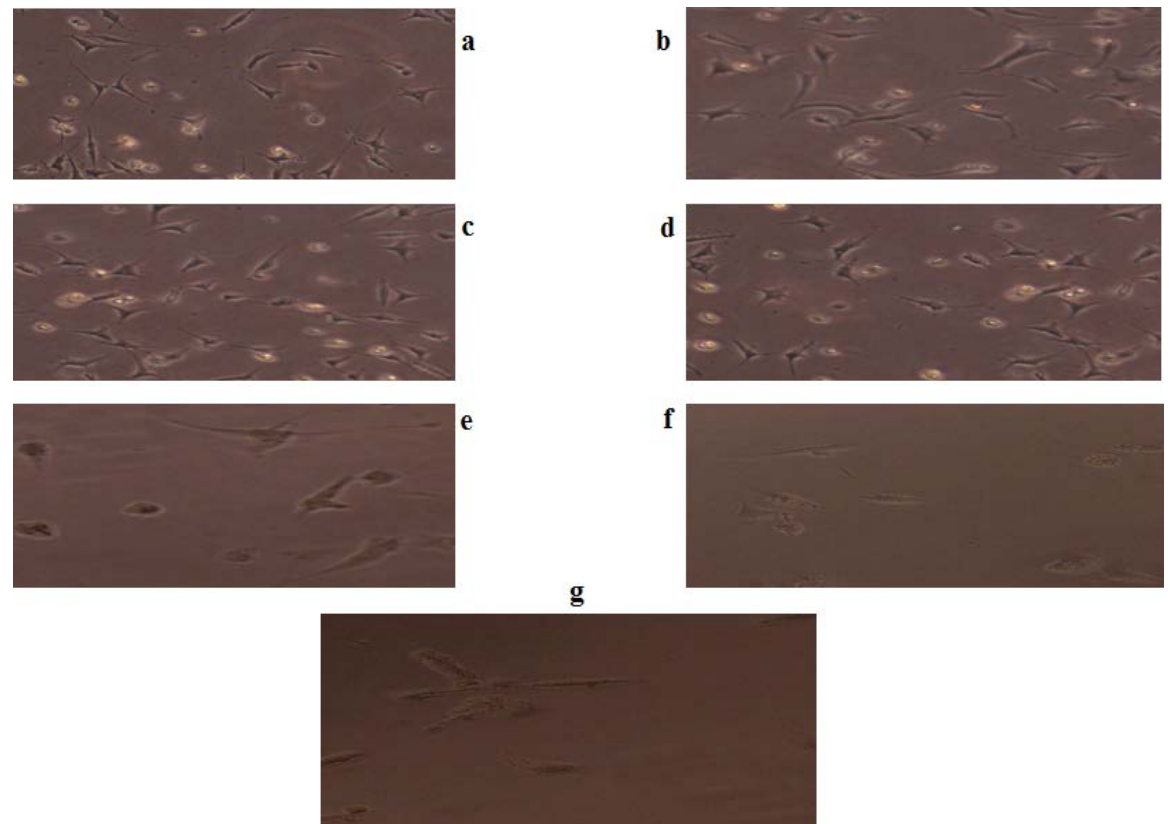

Fig. 7: Viability of cells treated with control and the test samples

a*: Control, b-d: 5, 10, 15\% of neem oil, respectively and e-g: 1, 2, $5 \%$ of APG emulsifier, respectively

topical agents, and also their SPFs are similar. Such compliance makes this green emollient formulations a very good substitute for loading cosmeceuticals and aiming for their dermal delivery. Further, the cream's basic constituent components are nontoxic to 3T3 skin lines and also to representative normal skin flora. This favourably addresses toxicity concerns, if any, to the usage of the polyglucoside-based emollient creams. Further studies, based on effective loading of other cosmeceuticals and pharmaceutically active ingredients onto this emollient cream and study/optimization of their in vitro and in vivo release rate kinetics is under progress with promising results.

\section{Acknowledgements:}

The authors thank VIT University, Vellore and Director, CSIR National Aerospace Laboratories, Bangalore for facilitating this study.

\section{Conflicts of interest:}

There are no conflicts of interest.

\section{Financial support and sponsorship:}

Nil.

\section{REFERENCES}

1. Prausnitz MR, Mitragotri S, Langer R. Current status and future potential of transdermal drug delivery. Nat Rev Drug Discov 2004;3:115-24.

2. Williams A. Transdermal and topical drug delivery. London: Pharmaceutical Press; 2003.

3. Parante MA, Gambaro A, Solana A. Study of sensory properties of emollients used in cosmetics and their correlation with physicochemical properties. J Cosmet Sci 2005;56:175-82.

4. Savary G, Grisel M, Picard C. Impact of emollients on the spreading properties of cosmetic products: A combined sensory and instrumental characterization. Colloids Surf B Biointerfaces 2013;102:371-78.

5. Fruijtier- Polloth C. Safety assessment on polyethylene glycols 
(PEGs) and their derivatives as used in cosmetic products. Toxicology 2005;214:1-38.

6. Aalto-Korte K, Pesonen M, Kuuliala O, Suuronen K. Occupational allergic contact dermatitis caused by coconut fatty acids diethanolamide. Contact Dermatitis 2014;70:169-74.

7. Thornfeldt C. Cosmeceuticals containing herbs: fact, fiction, and future. Dermatol Surg 2005;31:873-80.

8. Banerjee K, Thiagarajan N, Thiagarajan P. Azadirachta indica A. Juss based emollient cream for potential dermatological applications. Indian J Pharm Sci 2016;78:320-25.

9. Rice WR. Analyzing tables of statistical tests. Evolution 1989;43:223-25.

10. Shahavi MH, Hosseini M, Jahanshahi M, Meyer RL, Darzi GN. Evaluation of critical parameters for preparation of stable clove oil nanoemulsion. Arab J Chem 2015.

11. Krstonošić V, Dokić L, Nikolić I, Dapčević T, Hadnađev M. Influence of sodium dodecyl sulphate concentration on disperse and rheological characteristics of oil in water emulsions stabilized by OSA starch - SDS mixtures. J Serb Chem Soc 2012;77:83-94.

12. Barnes HA. Thixotropy a review. J Nonnewton Fluid Mech 1997;70:1-33.

13. Sudhahar V, Balasubramanian V. Sun production factor (SPF) determination of marketed sunscreen formulation by in-vitro method using UV-Vis spectrophotometer. Arch Appl Sci Res 2013;5:119-22.

14. Mansur JS, Breder MNR, Mansur MCA, Azulay RD. Determinagao Do Fator De Protegâo Solar Por Espectrofotometria. An Bras Dermatol Rio De Janeiro 1986;61:121-4.

15. https://www.asm.org/index.php/34-international/asmmeetings-and-conferences/274-antimicrobial-susceptibilitytesting-manual.

16. Bauer AW, Kibry WMM, Sherri JC, Turck M. Antibiotic susceptibility testing by a standardized single disc method. Am J Clin Pathol 1966;45:493-96.

17. Mosmann T. Rapid colorimetric assay for cellular growth and survival: application to proliferation and cytotoxicity assays. $\mathrm{J}$ Immunol Methods 1983;65:55-63.

18. Farrar JJ, Farrar JF, Simon PL, Hifliker ML, Stadler BM, Farrar WL. Thymoma production of $\mathrm{T}$ cell growth factor
(Interleukin 2). J Immunol Res 1980;125:2555-58.

19. Falco OB, Korting HC. Der Normale pH-Wert der Menschlichen Haut. Der Hautarzt 1986;37:126-29.

20. Sułek MW, Ogorzałek M, Wasilewski T, Klimaszewska E. Alkyl polyglucosides as components of water based lubricants. J Surfactants Deterg 2013;16:369-75.

21. Preziosi V, Perazzo A, Caserta S, Tomaiuolo G, Guido S. Phase inversion emulsification. Chem Eng Trans 2013;32:1585-90.

22. Kaltsa O, Gatsi I, Yanniotis S, Mandala I. Influence of ultrasonication parameters on physical characteristics of olive oil model emulsions containing xanthan. Food Bioprocess Technol 2014;7:2038-49.

23. Sautina NV, Sitdikova K I, Galyametdinov YuG. Study of phase transitions in lyotropic liquid-crystal emulsion systems tetraethylene glycol monododecyl ether, water, and vaseline oil by the wetting angle method. Russ J Appl Chem 2014;87:419-23.

24. Savic S, Lukic M, Jaksic I, Reichl S, Tamburic S, MüllerGoymann C. An alkyl polyglucoside-mixed emulsifier as stabilizer of emulsion systems: The influence of colloidal structure on emulsions skin hydration potential. J Colloid Interface Sci 2011;358:182-91.

25. Wiacek A, Chibowski E. Zeta potential, effective diameter and multimodal size distribution in oil:water emulsion. Colloids Surf A Physicochem Eng Asp 1999;159:253-61.

26. Cross MM. Rheology of non-Newtonian fluids: A new flow equation for pseudoplastic systems. J Colloid Sci 1965;20:417-37.

27. Lukic M, Jaksic I, Krstonosic V, Dokic L, Savic S. Effect of small change in oil phase composition on Rheological and textural properties of w/o emulsion. J Texture Stud 2013;44:34-44.

28. Ghannam MT, Hasan SW, Abu-Jdayil B, Esmail N. Rheological properties of heavy \& light crude oil mixtures for improving flowability. J Pet Sci Eng 2012;81:122-28.

29. Vianna-Filhoa RP, Petkowicza CLO, Silveira JLM. Rheological characterization of $\mathrm{O} / \mathrm{W}$ emulsions incorporated with neutral and charged polysaccharides. Carbohydr Polym 2013;93:266-72.

30. De Souza Mendes PR. Modeling the thixotropic behavior of structured fluids. J Nonnewton Fluid Mech 2009;164:66-75. 